\title{
THRESHOLD RADIUS OF BUDKER-CHIRIKOV INSTABILITY IN ELECTRON AND ION BEAMS WITH STRONGLY DIFFERENT RADII
}

\author{
Yu.Ya. Golub* \\ Moscow Radiotechnical Institute, 132 Warshavskoye shosse, 113519 Moscow, Russia
}

\begin{abstract}
The Budker-Chirikov instability (BCI) in a two-beam electron-ion system is researched. The BCI is due to the interaction of the slow cyclotron wave of the electron beam with the fast betatron wave of the ion beam. The electron-ion system consists of a high current relativistic electron beam propagating co-axially with a nonrelativistic ion beam in a drift tube. The external radii of the electron and ion beams are considered to be strongly different. The BCI increment decreases with the decrease in the ion beam radius when the electron beam radius is unchanged [1]. The $\mathrm{BCI}$ resonance frequency is shifted in this case. Existing the threshold ion beam radius, smaller than that radius BCI does not develop. Now, the cause of threshold radius existing is investigated.
\end{abstract}

\section{BASIC EQUATIONS}

We investigate a two-beam electron-ion system consisting of a nonrelativistic ion beam propagating coaxially with a high-current relativistic electron beam. The both beams are injected in equilibrium into drift tube. Beams radii can be differ each other in few times, unlike [2]. The kinetic description- of both beams is provided by means of solutions of the Vlasov equations for the electron and ion distributions functions, $f_{\mathrm{e}, \mathrm{i}}\left(\mathrm{t}, \mathrm{z}, \mathrm{r}, \mathrm{v}_{\mathrm{z}}, \mathrm{v}_{\mathrm{r}}\right.$, $\left.\mathrm{v}_{\theta}\right)$. The equations for the scalar potential and the three component of the vector potential are used for finding the electromagnetic fields. The equations are solved in the long-wave $\left(\partial^{2} / \partial \mathrm{z}^{2}<<\Delta_{\perp}\right)$, low-frequency $\left(\partial^{2} / \partial \mathrm{t}^{2}<<\mathrm{c}^{2}\right.$ $\left.\Delta_{\perp}\right)$, axial-symmetric $(\partial / \partial \theta \equiv 0)$ case. where $\Delta_{\perp}$ is the transverse part of the Laplace operator. Boundary conditions for the potentials fellow from the system's axial symmetry, the presence of conducting tube with radius $\mathrm{R}$ and the gauge condition $\operatorname{div} \boldsymbol{A}=0$. The Vlasov equations are solved by the macroparticle method. It is assumed that the steady-slate process is periodic in time set with a frequency $\omega$. In this case it is convenient to use the longitudinal coordinate $\mathrm{z}$ as the independent variable, using the relation $\mathrm{d} / \mathrm{dt}=\left(\mathrm{l} / \mathrm{v}_{\mathrm{z}}\right) \mathrm{d} / \mathrm{dz}$, where $\mathrm{v}_{\mathrm{z}}$, is the velocity of a given macroparticle. The problem is then reduced to the evolution of a periodic-in-time system on $\mathrm{z}$.

The periodic in time (with frequency w) potential function are of the form

$$
\mathrm{G}(\mathrm{t}, \mathrm{z}, \mathrm{r})=\overline{\mathrm{G}}(\mathrm{z}, \mathrm{r})+\operatorname{Re}\left\{\sum_{j} \tilde{\mathrm{G}}(\mathrm{z}, \mathrm{r}) \cdot \mathrm{e}^{\mathrm{ij \omega t}}\right\}
$$

which is substituted into the equations for the potential components and integrated over a time period. The equations for the four components of the 4-poternial $A_{i}$, ( $\Delta_{\mathrm{ri}} A_{i}=4 \pi \rho_{\mathrm{i}}, \mathrm{i}=1,2,3,4 \Delta_{\mathrm{ri}}$ is the radial parts of the Dalamber operator, and $\rho_{i}$ are the components of the 4density) are solved at every z--cross-section by the grid method.

\section{BCI INSTABILITY}

The important of the instabilities in the two-beam electron-ion system are the Budker-Chirikov instability (BCI) $[3,4]$. They are connected with the resonance of the slow-cyclotron wave of tile electron beam and the fast Langmure wave and fast betatron wave of the ion beam, respectively. Unlike [2], the external radii of the electron and ion beams are considered to be strongly different.

The case, where the ion beam radius less than the electron beam radius is investigated next. The BudkerChirikov instability take place in that case at some system parameters. The instability in the time periodic regime is displayed in the growth of the radial modulation amplitude of both beams along the longitudinal coordinate. Also the BCI is developed in exponential growth of the potential amplitude. The BCI increment decreases with the decrease in the ion beam radius when the electron beam radius is unchanged. The BCI resonance frequency is shifted in this case.

The dependence of space increment $\mathrm{E}$ of $\mathrm{BCI}$ on the ion beam radius ri is shown in Fig. 1. The electron beam current $\mathrm{J}=0.5 \mathrm{kA}$, ion longitudinal velocity $\mathrm{b}=0.03$, relativistic factor $\mathrm{g}=3$, longitudinal magnetic field $1 \mathrm{kG}$, the drift tube radius $\mathrm{R}=1.5 \mathrm{~cm}$, ion beam density $\mathrm{ni}=\mathrm{f}^{*} \mathrm{ne}, \mathrm{f}=1 / 9$, the electron beam radius $=0.6^{*} \mathrm{R}$. The resonance frequency is to the increment maximum. The ion beam radius is normalized on $\mathrm{R}$.

We see in Fig.1, that the BCI increment is decreased with decreasing of the ion beam radius. If the ion beam radius is equal to $0.1 * \mathrm{R}$ the $\mathrm{BCI}$ does not develop at distances up to $500 \mathrm{drift}$ tube radius $\mathrm{R}$. Increment is equal to 0 when the ion beam radius $0.1 * \mathrm{R}$.

*yurigolub@mtu-net.ru 


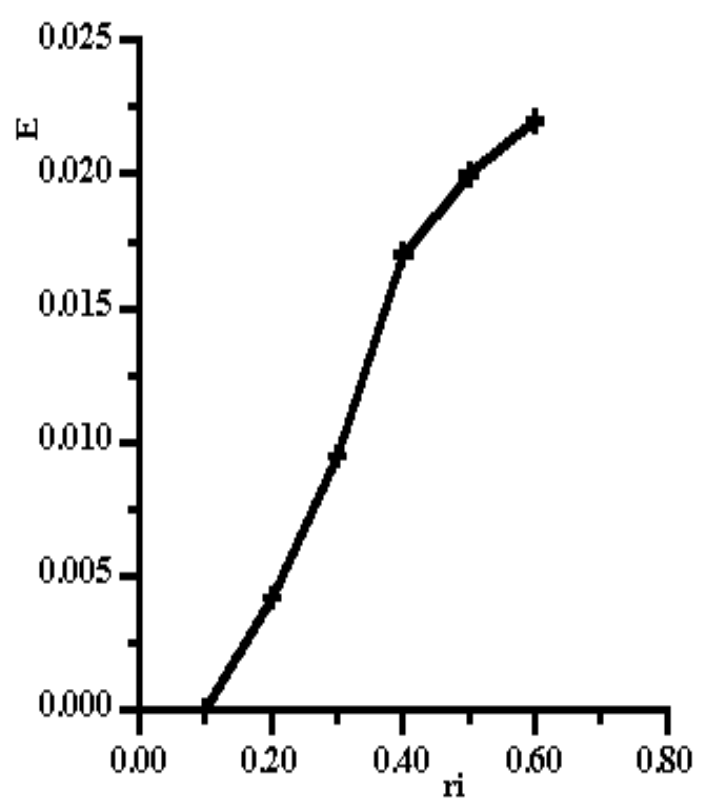

Fig.1: The dependence of space increment $\mathrm{E}$ of $\mathrm{BCI}$ on the ion beam radius ri.

The BCI saturation mechanism is associated with the change of the oscillation wavelength of the separate particles [2]. The equilibrium ion beam fields are nonlinear function of radius in the region outside of the ion beam. Thus in electron beam are existed two parts. First part of electron beam is oscillated in nonlinear fields outside the ion beam radius. Second part of electron beam is oscillated in linear fields inside the ion beam radius. Only second part is took part in BCI [1]. When the ion beam radius is decreased the second part of electron beam also is decreased. Therefore BCI increment is decreased. But the ions oscillation wavelength in the second part of electron beam changes due to an increase of nonisochronism with the amplitude growth, as in case with the beams with equal radius [2]. These speculation don't explain BCI threshold.

We see in Fig.2, that the increment line is two parabolas. First parabola occur for no small ion beam radius from 0.4 to 0.6 . $\mathrm{BCI}$ increment decrease cause is to change of amount of electron beam in BCI. We see, that BCI threshold no exist for first parabola. Second parabola is occurred for small ri from 0.1 to 0.4 . parabola is the case of small ion beam radius. Thus transient ion

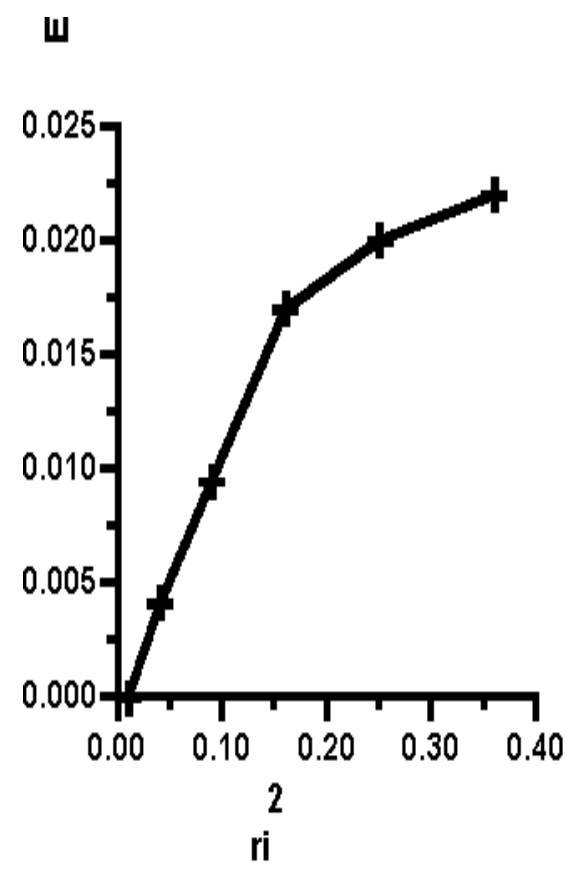

Fig.2: The dependence of space increment $\mathrm{E}$ of $\mathrm{BCI}$ on the ion beam radius ri to square.

beam radius between two parabolas is existed. Transient ion beam radius is equal to 0.4 for discussed above parameters. Only for second parabola BCI threshold is existed. The ion beam density become ununiform during oscillations. Ions oscillate in nonlinear fields. Therefore the change of the oscillation wavelength of the separate particles is occurred. Thus when ion beam radius very small the BCI does not exist.

\section{REFERENCES}

[1] Yu.Ya. Golub, EPAC Proceedings, 1125 (2000)

[2] Yu.Ya. Golub, N.E.Rozanov, Nuclear Instruments and Methods in Physics Research, A358 (1995) 479

[3] G.I. Budker. Atomnaya energiya 1 (1956) 9

[4] B.V. Chirikov, Atomnaya energiya 19 (1965) 239 